Meta

Journal des traducteurs

Translators' Journal

\title{
L'histoire d'une brève rencontre
}

\section{Anatole Sliosberg}

Volume 23, numéro 3, septembre 1978

URI : https://id.erudit.org/iderudit/004169ar

DOI : https://doi.org/10.7202/004169ar

Aller au sommaire du numéro

Éditeur(s)

Les Presses de l'Université de Montréal

ISSN

0026-0452 (imprimé)

1492-1421 (numérique)

Découvrir la revue

Citer cet article

Sliosberg, A. (1978). L'histoire d'une brève rencontre. Meta, 23(3), 195-199.

https://doi.org/10.7202/004169ar

Ce document est protégé par la loi sur le droit d'auteur. L'utilisation des services d'Érudit (y compris la reproduction) est assujettie à sa politique d'utilisation que vous pouvez consulter en ligne.

https://apropos.erudit.org/fr/usagers/politique-dutilisation/
Cet article est diffusé et préservé par Érudit.

Érudit est un consortium interuniversitaire sans but lucratif composé de l'Université de Montréal, l'Université Laval et l'Université du Québec à Montréal. Il a pour mission la promotion et la valorisation de la recherche. https://www.erudit.org/fr/ 


\section{L'histoire d'une brève rencontre}

Parmi les différents domaines dans lesquels s'exerce l'art de traduire, celui de la médecine et de la pharmacie est certainement l'un des plus vastes, mais aussi l'un des plus difficiles et des plus passionnants.

Il est évident qu'un traducteur travaillant dans le domaine médico-pharmaceutique doit posséder des notions précises sur le fonctionnement de l'organisme humain, sur les propriétés des substances pharmaceutiques et, enfin, sur une discipline relativement récente, la PHARMACOCINÉTIQUE(pharmacokinetics), consacrée à l'étude des mécanismes d'action et des modifications que subissent, lors de leur «brève rencontre", à la fois l'organisme humain et le médicament.

En ce qui a trait au domaine médical, le traducteur dispose de manuels de biologie et de physiologie qui lui décrivent le fonctionnement de l'organisme humain, et de dictionnaires de termes biologiques et médicaux qui lui permettent d'établir des équivalences. Il reste que les difficultés dans ce domaine sont multiples: les divergeances terminologiques (l'ULCĖRE GASTRO-DUODÉNAL devient peptic ulcer pour les Anglo-Saxons et l' ULCÈRE PEPTIQUE français se traduit en anglais par marginal ulcer ou stoma ulcer); les éponymies (la LOI DE MARIOTTE s'appelle Boyles'Law en Angleterre et en Amérique); les abréviations et les sigles ( $N A D$, non appreciable desease, se traduit en français par R.A.S., rien à signaler), etc.

Quant au domaine pharmaceutique, les difficultés sont moins d'ordre terminologique, elles résultent surtout de l'étendue des connaissances que ce domaine exige.

En effet, le très long chemin que parcourt le médicament, depuis sa découverte jusqu'à sa présentation finale destinée à être administrée au malade, intéresse à la fois la chimie, la botanique, la pharmacie galénique artisanale et surtout industrielle; cette dernière fait appel à un grand nombre de termes techniques appartenant aux domaines de la mécanique, de l'électricité, de l'électronique, de l'optique et, bien entendu, à l'ubiquitaire statistique; enfin les informations données par l'expérimentation, sur l'animal d'abord, puis sur les humains sélectionnés, s'exprime par une terminologie très touffue, propre à l'histologiste, au généticien, au zoologiste, au vétérinaire, au physiologiste et au pharmacologue.

Il nous est évidemment impossible de nous étendre sur tous ces aspects de la traduction pharmaceutique : nous nous bornerons à citer l'exemple de la fabrication d'un comprimé, qui parcourt un grand nombre d'étapes avant d'arriver sur la table de chevet du malade. 
L'étape chimique comprend l'extraction ou la synthèse d'une nouvelle substance et l'on sait que sur 5000 molécules découvertes ou mises au point par les chercheurs, il arrive souvent qu'une seule parvienne au stade d'utilisation sous forme de médicament. C'est dire la complexité de la "démarche de création" et la multiplicité des moyens mis en ouvre, que ce soit pour la synthèse ou pour l'extraction d'un PRINCIPE $\operatorname{ACTIF}$ (active ingredient) - un alcaloïde ou un glucoside par exemple - à partir d'une matière première végétale (ou "drogue», terme qu'il n'est pas permis de confondre avec le mot anglais "drug" désignant tout médicament d'origine naturelle ou synthétique).

Dès ce stade, le chercheur établit une sélection et de multiples contrôles d'identité et de pureté, en utilisant des méthodes purement chimiques ou physiques (la polarographie, la chromatographie, l'électrophorèse, la spectrophotométrie, etc.) qui possèdent chacune leur terminologie propre.

Une fois le produit défini dans sa structure et dans ses propriétés physiques et chimiques, commence la longue marche vers sa présentation en tant que médicament: on considère alors ses possibilités d'administration par différentes voies, et, par conséquent, avant tout, sa solubilité dans différents SOLVANTS(solvent), ses réactions acides ou alcalines, exprimées par les valeurs du $\mathrm{Ph}$ (Potentil d'Hydrogène), ses incompatibilités éventuelles, etc.

Dans le cas que nous avons choisi comme type, le produit réduit en poudre doit subir ce que l'on appelle une granulation, c'est-à-dire une opération permettant d'égaliser les particules et d'assurer ainsi une meilleure COULANCE(flowance) dans les tubulures des machines à comprimer; c'est à ce stade que le principe actif est additionné d'adjuvants, les uns pour favoriser la cohésion du futur comprimé : les AGGLUTINANTS ou LIANTS (ligant); d'autres pour faciliter son DELITEMENT (disintegration), d'autres encore, les EXCIPIENTS/ ou AGENTS DE LESTAGE (excipient ou filler), pour lui donner la consistance voulue et enfin les COLORANTS (dyestuff).

Le granulé qui résulte de cette MIXTION (mixing) va parcourir les différents segments de la machine à comprimer.

La TREMIE D'ALIMENTATION (feed-hopper), prolongée par un tuyau, conduit cette «MASSE TABLETTAIRE» (tabletting mass) vers un dispositif mobile spécial, le SABOT (feed-shoe), qui se porte en avant pour remplir la MATRICE(die), cavité creusée dans une tablette horizontale. Au même moment, deux POINÇONS (punch) pénètrent dans cette cavité pour comprimer le granulé pendant que le sabot recule. Une nouvelle avance de celui-ci repousse le comprimé formé et apporte une nouvelle charge à la matrice. Les mouvements sont synchronisés avec, cependant, un léger décalage pour le poinçon inférieur qui soulève le comprimé au-dessus du plan de la tablette, ce qui permet son balayage par le sabot. Un système d'ARBRES A CAME( cam shaft), relié à un moteur, assure le fonctionnement de l'ensemble de cette machine à comprimer.

Les comprimés sont acheminés vers un dispositif de comptage, de contrôle et de remplissage de tubes ou vers une machine qui les inclut dans les plaquettes en matière plastique. Divers appareils permettent de vérifier la conformité des produits de cette fabrication aux spécifications et aux normes établies : dimensions, temps de délitement, RESISTANCE Ȧ L'USURE (wear resistance), etc. 
La préparation des dragées parcourt, elle aussi, diverses étapes, dont la plus importante est l'ENROBAGE (coating) qui s'effectue dans les turbines.

Nous ne mentionnons que pour mémoire la fabrication des suppositoires, des solutions injectables, des pommades, etc.

Quelle que soit la forme médicamenteuse, le produit passera entre les mains des expérimentateurs et c'est à ce stade que se jouera son sort. Deux critères essentiels interviennent ici : l'ACTIVITÉ (activity) du médicament et son INNOCUITÉ (safety).

L'expérimentation se fait surtout sur diverses espèces animales : souris, rats, hamsters, lapins, chats, chiens, singes. De plus, on doit sélectionner certaines souCHES (strain) parmi l'espèce choisie, car on s'est aperçu que diverses lignées ne réagissent pas de manière identique à un seul et même produit; l'étude de ce phénomène fait l'objet d'une branche nouvelle de la pharmacologie: la PHARMACOGENETIQUE (pharmacogenetics).

Ajoutons que, depuis un certain nombre d'années, plus précisément depuis la tragédie de la Thalidomide, on cherche avec une attention toute particulière, les effets éventuels des nouveaux médicaments sur la descendance des animaux, et certains organismes officiels, chargés de réglementer l'admission de ces produits sur le marché, exigent une expérimentation très prolongée sur plusieurs générations d'animaux.

Outre l'expérimentation sur les animaux, on procède à des tests sur des CULTURES DE TISSUS ( issue culture), sur des cultures de microbes lorsqu'il s'agit de médicaments antimicrobiens ou, plus rarement, sur des cultures de parasites protozoaires.

L'étude de l'action du médicament sur l'animal envisage les effets recherchés sur les différents tissus et systèmes de l'organisme (appareils respiratoire et circulatoire, tube digestif, système nerveux, rein, fonctions de reproduction), mais ausi les EFFETS SECONDAIRES, indésirables (side effects) ou franchement dangereux, les REACTIONS DÉFAVORABLES (adverse reactions), qui attirent plus spécialement l'attention des chercheurs, en vertu du principe primum non nocere. Il en est de même des études toxicologiques qui permettent d'établir les doses que l'on ne doit pas dépasser sous peine de déclencher des phénomènes préjudiciables à la santé. Les DOSES UNITAIRES (individual doses) et les DOSES TOTALES(total doses) permettent d'établir la posologie (en anglais dosage, que l'on ne doit pas traduire dans ce contexte par dosage) du médicament, habituellement calculée par unité de poids de l'animal ou, dans certains cas, par unité de surface cutanée chez l'homme et, en particulier, chez l'enfant.

L'expérimentation se fait habituellement sur deux lots d'animaux, les uns atteints de la maladie à traiter, les autres normaux, servant de TÉMOINS (control animals).

Lorsque cette étape est franchie et que les résultats s'avèrent positifs, le médicament est confié à des cliniciens qui procèdent à une expérimentation sur l'homme. Signalons que, depuis plusieurs décennies, on utilise deux méthodes particulières qui assurent la FIABILITE (reliability) et le caractère objectif des constatations faites en clinique.

Il s'agit d'une part de l'emploi des "placebos» ou médicaments factices. On sait, en effet, que certains sujets, surtout lorsqu'ils sont atteints de maladies chroniques très 
éprouvantes, prennent parfois leurs espoirs pour des réalités et réagissent favorablement à tout nouveau produit prescrit par le médecin qui jouit de leur confiance et faussent ainsi, inconsciemment, les résultats de l'expérimentation.

D'autre part, la méthode A DOUBLE ANONYMAT ou À DOUBLE INSU (double blind test) permet d'éliminer, à la fois, l'éventualité d'une réponse trop favorable de la part du malade et un optimisme exagéré du médecin qui désire lui venir en aide: il s'agit de l'emploi alterné de deux formes médicamenteuses apparemment identiques mais qui, en réalité, contiennent l'une le médicament en étude et l'autre une substance pharmacologique inactive. Seul, le pharmacien qui les a préparées connaît la nature du produit effectivement pris par le patient et peut tirer des conclusions quant à l'activité réelle du nouveau produit, grâce à une étude statistique précise.

Nous avons esquissé l'histoire naturelle d'un médicament et indiqué les étapes de sa mise au point et les essais auxquels il est soumis. Il reste à envisager ce qui se passe lors de la «brève rencontre» entre l'organisme humain et le médicament.

En fait, il s'agit d'une conjonction entre un élément immuable, normalisé, toujours identique à lui-même - le médicament - et un être vivant, mouvant, soumis à des influences multiples en fonction du sexe, du code génétique, de l'âge, de l'état physique, de l'état physiologique, de l'environnement (climat, latitude). De plus, certains facteurs d'agression, comme les microbes, sont eux-mêmes variables dans leurs propriétés pathogènes. On conçoit donc l'extraordinaire complexité des problèmes qui surgissent lorsqu'on entreprend l'étude pharmaco-clinique d'un nouveau produit et la richesse de la terminologie impliquée.

Il faut savoir qu'avant d'agir, le médicament doit être libéré de la forme sous laquelle il est administré. Cette LIBERATION (release) assurera la DISPONIBILITÉ PHYSIOLOGIQUE(bioavailability), c'est-à-dire l'aptitude de l'organisme à disposer de la substance administrée. Cette libération peut être immédiate et permettre ainsi une action rapide du médicament, ou retardée grâce à la mise en œuvre de techniques de préparations spéciales comme l'usage de comprimés à couches multiples. On peut aussi combiner, dans un seul et même comprimé ou dans une gélule, l'action de deux médicaments, l'un assurant l'endormissement et l'autre procurant un sommeil plus prolongé. Enfin l'utilisation d'excipients ou de véhicules particuliers permet une LIBERATION PROLONGEE (sustained release) du principe actif.

Le médicament doit ensuite traverser divers barrages physiologiques, la paroi du tube digestif notamment. Sa RÉPARTITION (distribution) dans les organes ou dans les tissus se fait par voie sanguine, le principe actif circulant soit sous forme libre en ETAT DE DISSOLUTION(solubilized state), soit fixé sur les protéines du plasma, cette dernière forme étant souvent moins active que la première. Il existe cependant des barrières que certains médicaments ne peuvent franchir : il en est ainsi de la BARRIERE HEMATOENCÉPHALIQUE ou HEMOENCÉPHALIQUE (blood-brain barrier) qui s'oppose à la pénétration de certains produits dans le système nerveux à partir du sang; de la BARRIÈRE PLACENTAIRE (placental barrier) qui protège le fœtus des substances circulant dans le sang de la mère. 
Au cours de ses pérégrinations, le médicament va subir, principalement sous l'influence des enzymes propres aux différents organes et humeurs de l'organisme, des transformations qui le décomposeront en substances plus simples dites METABOLITES (metabolite), susceptibles de pénétrer dans l'intimité des tissus et des cellules. Ces transformations sont désignées sous le terme de METABOLISME.

Les réactions enzymatiques, pour leur part, peuvent être inhibées par certains produits ou activitées par d'autres. Ainsi, lorsque les enzymes spécifiques sont inhibées, le métabolisme des médicaments concernés est ralenti, leur action générale affaiblie ou retardée. $\mathrm{Si}$, au contraire, les enzymes sont exaltées dans leurs effets, les transformations sont accélérées et augmentées et l'effet des médicaments s'en trouve accru. D'autres données peuvent aussi jouer un rôle important: l'âge, le sexe, l'espèce, la race, etc. Enfin, parmi les facteurs modifiant le métabolisme des médicaments, on doit signaler les interactions médicamenteuses. Certaines actions conjuguées des médicaments peuvent être bénéfiques, soit grâce à l'addition des effets des divers éléments, soit par suite de SYNERGIE(synergism) caractérisée par le fait que les effets de deux substances données simultanément sont plus marqués que la somme des effets isolés de chacune d'elles, soit encore grâce à la POTENTIALISATION (potentialization ou potentiation), c'est-à-dire le renforcement de l'action du médicament sous l'influence d'un autre produit ; ces deux derniers phénomènes - synergie et potentialisation - s'opposant à l'antagonisme qui consiste en la suppression de l'action d'un produit par une autre substance.

Il nous reste à envisager le dernier acte de cette «brève rencontre" : l'excrétion.

Les médicaments suivent la même voie que les produits endogènes: ils s'éliminent surtout par voie urinaire. Mais ils sont aussi excrétés par voie fécale, biliaire, sudorale et par le lait de la mère.

Qu'arrive-t-il lorsque les deux protagonistes de cette rencontre se séparent?

Le médicament, pour sa part, est éliminé soit sous sa forme inchangée, soit sous celle de l'un ou de plusieurs de ses métabolites. La destinée de l'organisme humain est évidemment plus intéressante à suivre. Plusieurs cas peuvent se présenter :

a) Malgré l'intervention du médicament, rien n'est modifié : il s'agit d'un échec du traitement et la maladie continue son EvOLUTION (progression).

b) Le médicament a été efficace (effective) et a réussi

- soit à détruire le germe pathogène;

- soit à combler le déficit d'une carence;

- soit à rétablir le fonctionnement normal d'un organe ou d'un processus d'ordre nutritif.

c) Le médicament s'est avéré délétère, provoquant des effets secondaires ou des réactions défavorables ou encore des effets TERATOGENES (teralogenic effects).

En définitive, cette rencontre entre deux éléments, l'un immuable et normalisé le médicament - et l'autre mouvant, soumis à des influences multiples - l'organisme - quoique brève, n'a rien de simple ni pour le thérapeute... ni pour le traducteur.

DR ANATOLE SLIOSBERG avec la collaboration d'A mal Jammal 